\title{
The Jump Behavior of Foreign Exchange Market: Analysis of Thai Baht
}

\author{
Jow-Ran Chang \\ Department of Quantitative Finance, National Tsing Hua University \\ No. 101, Sec. 2, Kuang-Fu Rd., Hsinchu, Taiwan \\ jrchang@mx.nthu.edu.tw
}

\section{Mao-Wei Hung}

College of Management, National Taiwan University

No. 1, Sec. 4, Roosevelt Road, Taipei, Taiwan

hung@management.ntu.edu.tw

\section{Cheng-Few Lee}

Rutgers Business School, Rutgers University

New Jersey, USA

lee@rbsmail.rutgers.edu

\section{Hsin-Min Lu}

Eller College of Management, Univeristy of Arizona

Tucson, USA

hmlu@arizona.edu

We use square root stochastic volatility with or without jump model to study the heteroskedasticity and jump behavior of the Thai Baht. Bayesian factor is used to evaluate the explanatory power of competing model. It turns out that the square root stochastic volatility model with independent jump in observation and state equations (SVIJ) has the best explanatory power to our sample. Using the estimation results of the SVIJ model, we are able to link the major events of the Asian financial crisis to the jump behavior of either volatility or observation.

Keywords: Asian financial crisis; foreign exchange market; jump behavior; Markov chain Monte Carlo; stochastic volatility. 
- Jow-Ran Chang et al.

\section{Introduction}

There are two contributing factors to the exchange rate crises in developing countries. The first one is the behavior of the central bank and the second one is the strength of foreign exchange reserves. Before 1997, the central bank of Thai adopted a high interest rate and stable exchange rate policy; Thai baht was pegged to the US dollar. However, due to the high interest rate, a lot of capital flowed into Thai, which resulted in the bubble of speculative markets. When international speculators attacked Thai's financial market and there were insufficient foreign exchange reserves, the stable exchange rate policy collapsed. Therefore, understanding volatility dynamic is important to the realization the story behind the 1997 Asia financial crisis.

The Asian financial crisis was caused by speculators attacking Thai Baht and other Asian countries' currencies. The situation of Thai Baht started deteriorating in May 1997, but only started to receive public attention after Thai Baht depreciated $17 \%$ on July 2, 1997. Thailand's government turned to the IMF and other countries, including Japan and US for help. While Thailand's government was trying to deal with its financial puzzle, the crisis spread out and affected other south-east Asian countries. Thai Baht did not stop depreciating until early January, 1998.

Before May 1997, Thai Baht was pegged to the US dollar and seldom had a daily change of more than $1 \%$. From Fig. 1, we can see that Thai Baht's volatility increased substantially in May 1997, following a sudden depreciation of $17 \%$ on July 2 . The standard deviation of daily change in

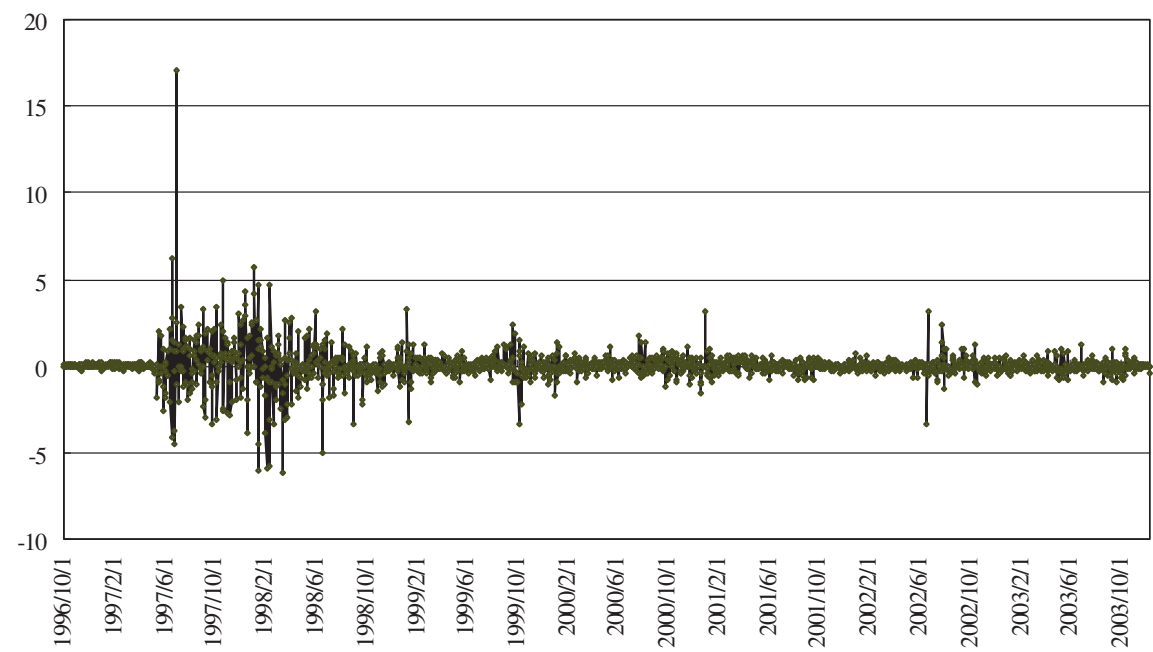

Fig. 1. Daily change rate of Thai Baht. 
May and June was $1.87 \%$, thus a $17 \%$ change was a realization of 9 standard deviations. Although a radical change like what happened on July 2 was very rare in the foreign exchange market, it does have important implications. This kind of market change may heavily increase foreign exchange risk and cause option to default. The above observation provides us with important guidelines as to how to model the behavior of the Thai Baht. First, the volatility at different time periods should be different. Second, even though the heteroskedasticity of exchange rate is being considered, some observations should be considered as outliers. Third, time series must implicitly model these extreme values (so called "jump") in order to reflect the full characteristics of the foreign exchange time series.

Many authors try to include heteroskedasticity and jump in time series models. For instance, Merton (1976a, b), Bates (1996), Bakshi, Cao and Chen (1997) consider option pricing with jump. Duffie and Kan (1995), Bjork, Kabanov and Rungaldier (1997), Das (1998), on the other hand, deal with jump problems in the bond market. Based on the Stochastic Volatility (SV) model and Markov Chain Monte Carlo (MCMC) method first proposed by Jacquier, Polson and Rossi (1994), Chib, Nardari and Shephard (2002) and Eraker, Johannes and Polson (2003) estimate a Stochastic Volatility model with jump using Observation Equation (SVJ). Further more, Eraker, Johannes and Polson (2003) estimate a SV model with jump components both in the observation and state equations. Jump behavior in the US stock market is clearly identified in Eraker, Johannes and Polson (2003). They found that jump in return and volatility significantly increases the curvature of implied volatility curves. Bayesian factors are used in this study to confirm the speculation that time series models with jump have better explanatory power than index return data.

The models used in our study are Log Stochastic Volatility (Log SV) and Square Root Stochastic Volatility model with or without jump in observation and state equations. The MCMC (Markov Chain Monte Carlo) method is used to estimate the models. Compared to other estimation methods, MCMC has three advantages (Johannes and Polson, 2002). First, MCMC estimates state variable (volatility and jump size in our models) as a byproduct when conducting estimation. Second, MCMC accounts for estimation risk. Third, MCMC requires only conditional posterior distributions of parameters, which is much easier to derive than an unconditional posterior distribution as required in other likelihood function based methods.

The rest of the article is arranged as follows. Section 2 introduces and derives the Square Root SV model and Section 3 summarizes the empirical 
results. Model diagnose is given in Section 4 and a discussion of how the estimated jump behavior can be connected to the events of the Asian financial crisis is in Section 5. Conclusion is presented in Section 6.

\section{Stochastic Volatility Model with Jumps}

\subsection{Model}

A Square Root Volatility can be written as

$$
\begin{aligned}
& r_{t}=d+\sqrt{h_{t-1}} \varepsilon_{t} \\
& h_{t}=\alpha+\delta h_{t-1}+\sqrt{h_{t-1}} u_{t} .
\end{aligned}
$$

The first equation in Eq. (1) is called observation equation or level equation, and the second one is state equation or transition equation. At a given time $t$, the observation equation shows that the change of exchange rate follows a normal distribution with mean $d$ and variance $h_{t-1}$. The state equation, on the other hand, describes how the state variable $h_{t}$ evolves through time. The variance for the next period is serially correlated with that of the current period. The covariance matrix of $\varepsilon_{t}$ and $u_{t}$ is

$$
\operatorname{var}\left(e_{t}\right) \equiv \Sigma \equiv\left(\begin{array}{cc}
1 & \rho \sigma_{\nu} \\
\rho \sigma_{\nu} & \sigma_{\nu}^{2}
\end{array}\right)
$$

where $e_{t} \equiv\left(\varepsilon_{t}, u_{t}\right)^{\prime}$.

If there is an asymptotic relationship in the time series, that is, the rise or fall of level equation will affects the realization of the state variable, and the correlation coefficient will not be zero. The correlation coefficient between the residual of observation and state equation is a measure of the leverage effect. In some financial time series, leverage effect plays a significant role. For example, the leverage effect in the US stock market is about -0.3 to -0.5 (Jacquier, Polson and Rossi, 2003).

If we add a jump component into the level equation, the model can be expressed as

$$
\begin{aligned}
& r_{t}=d+\sqrt{h_{t-1}} \varepsilon_{t}+\xi_{t}^{r} J_{t}^{r} \\
& h_{t}=\alpha+\delta h_{t-1}+\sqrt{h_{t-1}} u_{t} .
\end{aligned}
$$

The above model is called stochastic volatility with jump model (SVJ). The jump component $\xi_{t}^{r} J_{t}^{r}$ is a product of $J_{t}^{r}$, which controls whether the jump occured, and $\xi_{t}^{r}$, controls the size of the jump. $J_{t}^{r}$ follows a Bernoulli distribution with $\operatorname{pr}\left(J_{t}^{r}=1\right)=\lambda_{r}$. $\xi_{t}^{r}$ is a normal random variable with mean $u_{r}$ and variance $\sigma_{r}^{2}$. 
Moving one step further, the jump component can be added into the state equation. If the jump in level and state equations are independent, we will get a stochastic volatility model with independent jump at level and state equations (SVIJ). The model can be written as follows:

$$
\begin{aligned}
& r_{t}=d+\sqrt{h_{t-1}} \varepsilon_{t}+\xi_{t}^{r} J_{t}^{r} \\
& h_{t}=\alpha+\delta h_{t-1}+\sqrt{h_{t-1}} u_{t}+\xi_{t}^{v} J_{t}^{v}
\end{aligned}
$$

where $J_{t}^{v}$ also follows a Bernoulli distribution with $\operatorname{pr}\left(J_{t}^{v}=1\right)=\lambda_{v}$. To insure positive jump in state equation, $\xi_{t}^{v}$ follows an exponential distribution with mean $u_{v}$. With both jump components in the level and state equations, the SVIJ is the most flexible model.

Another possible setting is when jumps in level and state equations happen simultaneously, which is called stochastic volatility model with contemporaneous jump at level and state equations (SVCJ.) In this setting, $J_{t}^{r}=J_{t}^{v}$ and the jump size in the level and state equations is correlated with correlation coefficient $\rho_{J}$. The relation can be expressed as follows:

$$
\begin{aligned}
\xi_{t}^{v} & \sim \exp \left(u_{v}\right) \\
\xi_{t}^{r} \mid \xi_{t}^{v} & \sim N\left(u_{r}+\rho_{J} \xi_{t}^{v}, \sigma_{r}^{2}\right) .
\end{aligned}
$$

In our study, the log stochastic volatility model (Log SV) is also used for the purpose of comparison. The model can be expressed as follows:

$$
\begin{aligned}
r_{t} & =d+\sqrt{h_{t}} \varepsilon_{t} \\
\ln h_{t} & =\alpha+\delta \ln h_{t-1}+u_{t} .
\end{aligned}
$$

The covariance matrix of $\left(\varepsilon_{t}, u_{t}\right)^{\prime}$ is the same as the Square Root SV model. The difference lays in the state equation. In order to ensure that volatility is positive, Log SV model uses log to transform the equation. The log transformation also makes the state equation more flexible. A detail discussion on the subject can be found in Jacquier, Polson and Rossi (2003).

\subsection{The prior and posterior distribution}

MCMC (Markov Chain Monte Carlo) is used to estimate our models. The posterior distributions of parameters are needed to conduct simulation. Since SVJ, SVIJ and SVCJ have similar structures, the following discussion will only focus on the SVIJ model. A similar discussion can be found in Eraker, Johannes and Polson (2003).

The parameters that need to be estimated are $\theta=\left(d, \alpha, \delta, \rho, \sigma_{v}, u_{v}\right.$, $\left.u_{r}, \sigma_{r}^{2}, \lambda_{r}, \lambda_{v}\right)^{\prime}$ plus volatility of each period $h=\left(h_{1}, h_{2}, \ldots, h_{t}\right)^{\prime}$ and jump 
Jow-Ran Chang et al.

related latent variables $J_{r}=\left(J_{1}^{r}, J_{2}^{r}, \ldots, J_{T}^{r}\right)^{\prime}, J_{v}=\left(J_{1}^{v}, J_{2}^{v}, \ldots, J_{T}^{v}\right)^{\prime}$, $\xi_{r}=\left(\xi_{1}^{r}, \xi_{2}^{r}, \ldots, \xi_{T}^{r}\right)^{\prime}$ and $\xi_{v}=\left(\xi_{1}^{v}, \xi_{2}^{v}, \ldots, \xi_{T}^{v}\right)^{\prime}$.

First, the jump status $J_{r}, J_{v}, \xi_{r}, \xi_{v}$ can be taken as given, and the model arranged as

$$
\begin{aligned}
& \tilde{r}_{t} \equiv r_{t}-\xi_{t}^{r} J_{t}^{r}=d+\sqrt{h_{t-1}} \varepsilon_{t} \\
& \tilde{h}_{t} \equiv h_{t}-\xi_{t}^{v} J_{t}^{v}=\alpha+\delta h_{t-1}+\sqrt{h_{t-1}} u_{t} .
\end{aligned}
$$

Thus we have

$$
\left(\begin{array}{c}
\frac{\tilde{r}_{t}-d}{\sqrt{h_{t-1}}} \\
\frac{\tilde{h}_{t}-\alpha-\delta h_{t-1}}{\sqrt{h_{t-1}}}
\end{array} \mid \theta, h_{t-1}, \xi_{t}^{r}, \xi_{t}^{v}, J_{t}^{r}, J_{t}^{v}\right) \sim N\left(\left(\begin{array}{l}
0 \\
0
\end{array}\right), \Sigma\right) .
$$

Applying a change of variable technique, we get

$$
\begin{aligned}
& p\left(\tilde{r}_{t}, \tilde{h}_{t} \mid \theta, h_{t-1}, \xi_{t}^{r}, \xi_{t}^{v}, J_{t}^{r}, J_{t}^{v}\right) \\
& \quad \propto h_{t-1}^{-1} p\left(\frac{\tilde{r}_{t}-d}{\sqrt{h_{t-1}}}, \frac{\tilde{h}_{t}-\alpha-\delta h_{t-1}}{\sqrt{h_{t-1}}} \mid \theta, h_{t-1}, \xi_{t}^{r}, \xi_{t}^{v}, J_{t}^{r}, J_{t}^{v}\right) .
\end{aligned}
$$

Let $\tilde{r}=\left\{\tilde{r}_{1}, \tilde{r}_{2}, \ldots, \tilde{r}_{T}\right\}, \tilde{h}=\left\{\tilde{h}_{1}, \tilde{h}_{2}, \ldots, \tilde{h}_{T}\right\}$, then

$$
\begin{aligned}
p\left(\tilde{r}, \tilde{h} \mid \theta, \xi^{r}, \xi^{v}, J\right) & =p\left(\tilde{r}_{1}, \tilde{h}_{1} \mid \bullet, h_{0}\right) p\left(\tilde{r}_{2}, \tilde{h}_{2} \mid \bullet, h_{1}\right) \cdots p\left(\tilde{r}_{T}, \tilde{h}_{T} \mid \bullet, h_{T-1}\right) \\
& \propto \prod_{t=1}^{T} p\left(\frac{\tilde{r}_{t}-d}{\sqrt{h_{t-1}}}, \frac{\tilde{h}_{t}-\alpha-\delta \tilde{h}_{t-1}}{\sqrt{h_{t-1}}} \mid \bullet, h_{t-1}\right) \\
& \propto \prod_{t=1}^{T}|\Sigma|^{-1 / 2} e^{-\frac{1}{2} \operatorname{tr}\left(e_{t} \Sigma^{-1} e_{t}^{\prime}\right)} \\
& \propto|\Sigma|^{-T / 2} e^{-\frac{1}{2} \operatorname{tr}\left(\Sigma^{-1} A\right)}
\end{aligned}
$$

where $A=\Sigma_{t=1}^{T} e_{t} e_{t}^{\prime}$.

To facilitate the estimation of covariance matrix $\Sigma$, we transform from $\left(\rho, \sigma_{v}\right)$ to $(\psi, \Omega)$. That is,

$$
\Sigma=\left(\begin{array}{cc}
1 & \rho \sigma_{\nu} \\
\rho \sigma_{\nu} & \sigma_{\nu}^{2}
\end{array}\right)=\left(\begin{array}{cc}
1 & \psi \\
\psi & \psi^{2}+\Omega
\end{array}\right) .
$$

Since $|\Sigma|=\Omega$, we have $\Sigma^{-1}=\frac{1}{\Omega}\left(\begin{array}{cc}\psi^{2} & -\psi \\ -\psi & 1\end{array}\right)+\left(\begin{array}{ll}1 & 0 \\ 1 & 0\end{array}\right) \equiv \frac{C}{\Omega}+\left(\begin{array}{ll}1 & 0 \\ 0 & 0\end{array}\right)$. Let $a_{i j}$ be the $i j$ th element in matrix A, we have $\operatorname{tr}\left(\Sigma^{-1} A\right)=\operatorname{tr}(C A) / \Omega+a_{11}$. 
As a result,

$$
\begin{aligned}
p(\psi, \Omega \mid \tilde{r}, \tilde{h}, d, \alpha, \delta) & \propto \frac{1}{\Omega^{T / 2}} e^{-\frac{\operatorname{tr}(C A)}{2 \Omega}} \operatorname{pri}(\psi, \Omega) \\
& \propto \frac{1}{\Omega^{T / 2}} e^{-\frac{a_{22.1}+(\psi-\hat{\psi})^{2} a_{11}}{2 \Omega}} \operatorname{pri}(\psi, \Omega)
\end{aligned}
$$

where $a_{22.1}=a_{22}-\frac{a_{12}^{2}}{a_{11}}, \hat{\psi}=\frac{a_{12}}{a_{11}}$.

We assume a normal prior for $\psi$ and an inverse gamma for $\Omega$, that is

$$
\operatorname{pri}(\psi, \Omega)=\operatorname{pri}(\psi \mid \Omega) \operatorname{pri}(\Omega) \propto \exp \left(-\frac{\left(\psi-\psi_{0}\right)^{2}}{2 \Omega / p_{0}}\right) \Omega^{-\left(v_{0}+1\right)} \exp \left(\frac{-\lambda_{0}}{\Omega}\right) .
$$

Combining the last equation with Eq. (5), we get the posterior

$$
\begin{aligned}
p(\psi, \Omega \mid \tilde{r}, \tilde{h}, d, \alpha, \delta) & \\
& =p(\psi \mid \Omega, \tilde{r}, \tilde{h}, d, \alpha, \delta) p(\Omega \mid \tilde{r}, \tilde{h}, d, \alpha, \delta) \\
& =p(\tilde{r}, \tilde{h} \mid \psi, \Omega, d, \alpha, \delta) \operatorname{pri}(\psi \mid \Omega) p(\tilde{r}, \tilde{h} \mid \Omega, d, \alpha, \delta) \operatorname{pri}(\Omega) \\
& \propto \frac{1}{\Omega^{T / 2}} \exp \left(-\frac{a_{22.1}+(\psi-\hat{\psi})^{2} a_{11}}{2 \Omega}\right) \exp \left(\frac{\left(\psi-\psi_{0}\right)^{2}}{2 \Omega / p_{0}}\right) \Omega^{-\left(v_{0}+1\right)} \exp \left(\frac{-\lambda_{0}}{\Omega}\right) \\
& \propto \exp \left(-\frac{(\psi-\tilde{\psi})^{2}}{2 \Omega /\left(p_{0}+a_{11}\right)}\right) \Omega^{-\left(v_{0}+T / 2+1\right)} \exp \left(\frac{-\left(\lambda_{0}+a_{22.1} / 2\right)}{\Omega}\right)
\end{aligned}
$$

where $\tilde{\psi}=\left(a_{11} \hat{\psi}+p_{0} \psi_{0}\right) /\left(a_{11}+p_{0}\right)$. The result can be summarized as the following:

Prior distribution:

$$
\begin{aligned}
\operatorname{pri}(\Omega) & \sim I G\left(v_{0}, \lambda_{0}\right) \\
\operatorname{pri}(\psi \mid \Omega) & \sim N\left(\psi_{0}, \Omega / p_{0}\right) .
\end{aligned}
$$

Posterior:

$$
\begin{gathered}
p(\Omega \mid \tilde{r}, \tilde{h}, d, \alpha, \delta) \sim I G\left(v_{0}+T / 2, \lambda_{0}+a_{22.1} / 2\right) \\
p(\psi \mid \Omega, \tilde{r}, \tilde{h}, d, \alpha, \delta) \sim N\left(\tilde{\psi}, \Omega /\left(a_{11}+p_{0}\right)\right)
\end{gathered}
$$

The posterior of $\alpha$ and $\delta$ follows standard time series estimation. We assume normal prior for $(\alpha, \delta)$, that is $(\alpha, \delta)^{\prime} \sim N\left(\beta_{0}, \sigma_{\beta_{0}}^{2}\right)$, where $\beta_{0}$ is a $2 \times 1$ vector, and $\sigma_{\beta_{0}}^{2}$ is a $2 \times 2$ matrix. Since $\varepsilon_{t}$ and $u_{t}$ are correlated, we have the following relation $u_{t}=\sigma_{v} \rho \varepsilon_{t}+b_{2, t}, b_{2, t} \sim N\left(0,\left(1-\rho^{2}\right) \sigma_{v}^{2}\right)$. 
Let $y_{h}=\left(\frac{\tilde{h}_{2}}{\sqrt{h_{1}}}-\rho \sigma_{v} \varepsilon_{2}, \frac{\tilde{h}_{3}}{\sqrt{h_{2}}}-\rho \sigma_{v} \varepsilon_{3}, \ldots, \frac{\tilde{h}_{T}}{\sqrt{h_{T-1}}}-\rho \sigma_{v} \varepsilon_{T}\right)^{\prime}$ and $x_{h}=$ $\left(x_{h, 1}, x_{h, 2}, \cdots x_{h, T-1}\right)^{\prime}$, where $x_{h, t}=\left(\frac{1}{\sqrt{h_{t}}}, \frac{h_{t}}{\sqrt{h_{t}}}\right)^{\prime}$. The estimation of $(\alpha, \delta), \hat{\beta}$, is

$$
\hat{\beta}=\left(x_{h}^{\prime} x_{h}\right)^{-1} x_{h}^{\prime} y_{h}, \operatorname{var}(\hat{\beta})=\left(x_{h}^{\prime} x_{h}\right)^{-1} \sigma_{v}^{2}\left(1-\rho^{2}\right) .
$$

The posterior of $(\alpha, \delta)$ is $\left(\begin{array}{c}\alpha \\ \delta\end{array} \mid \tilde{h}, d, \rho, \sigma_{v}\right) \sim N(\tilde{\beta}, \tilde{V})$ where $\tilde{V}=\operatorname{var}(\hat{\beta})^{-1}+$ $\left(\sigma_{\beta_{0}}^{2}\right)^{-1}$ and $\tilde{\beta}=\tilde{V}\left(\operatorname{var}(\hat{\beta})^{-1} \hat{\beta}+\left(\sigma_{\beta_{0}}^{2}\right)^{-1} \beta_{0}\right)$.

The posterior distribution of $d$ can be derived in a similar fashion. From the relation between $u_{t}$ and $\varepsilon_{t}$, we have

$$
\varepsilon_{t}=\frac{\rho}{\sigma_{v}} u_{t}+b_{1 t}
$$

where $u_{t}$ and $b_{1 t}$ are independent, and $b_{1 t} \sim N\left(0,1-\rho^{2}\right)$.

Since we have $\tilde{r}_{t}=d+\sqrt{h_{t-1}} \varepsilon_{t}$, we plug in the relation of $u_{t}$ and $\varepsilon_{t}$. The equation can be written as

$$
\left(\frac{\tilde{r}_{t}}{\sqrt{h_{t-1}}}-\frac{\rho}{\sigma_{v}} u_{t}\right)=\frac{1}{\sqrt{h_{t-1}}} d+b_{1 t} .
$$

Let $y_{r}=\frac{\tilde{r}_{t}}{\sqrt{h_{t-1}}}-\frac{\rho}{\sigma_{v}} u_{t}$ and $x_{r}=\frac{1}{\sqrt{h_{t-1}}}$, then $\hat{d}=\left(x_{r}^{\prime} x_{r}\right)^{-1} x_{r}^{\prime} y_{r}, \operatorname{var}(\hat{d})=$ $\left(x_{r}^{\prime} x_{r}\right)^{-1}\left(1-\rho^{2}\right)$. Assuming we have normal prior for $d$ with mean $d_{0}$ and variance $\sigma_{d_{0}}^{2}$, then we have posterior distribution $d \sim N\left(\tilde{d}, \tilde{V}_{d}\right)$, where $\tilde{V}_{d}=$ $\operatorname{var}(\hat{d})^{-1}+\left(\sigma_{d_{0}}^{2}\right)^{-1}$ and $\tilde{d}=\tilde{V}_{d}\left(\operatorname{var}(\hat{d})^{-1} \hat{d}+\left(\sigma_{d_{0}}^{2}\right)^{-1} d_{0}\right)$.

We use a single move sampler to update $h_{t}$. That is, given other state variables and parameters, at time $t$, the posterior of volatility $h_{t}$ is derived. As time shifts forward, it will continue to update the reset volatility state variables. Random walk metropolis algorithm is used to sample the posterior of $h_{t}$. Note that there is a jump component in the state equation, and we only update the $h_{t}$ and take the jump size as given. To do this, we will first rewrite Eq. (4) as the following:

$$
\begin{aligned}
\tilde{r}_{t} & \equiv r_{t}-\xi_{t}^{r} J_{t}^{r}=d+\sqrt{h_{t-1}} \varepsilon_{t} \\
\tilde{h}_{t} & \equiv h_{t}-\xi_{t}^{v} J_{t}^{v}=\alpha+\delta h_{t-1}+\sqrt{h_{t-1}} u_{t}
\end{aligned}
$$

where $\tilde{h}_{t} \equiv h_{t}-\xi_{t}^{v} J_{t}^{v}$ is the jump non-related part of $h_{t}$.

At time $t+1$, we have

$$
\begin{aligned}
& \tilde{r}_{t+1} \equiv r_{t+1}-\xi_{t+1}^{r} J_{t+1}^{r}=d+\sqrt{\tilde{h}_{t}+\xi_{t}^{v} J_{t}^{v}} \varepsilon_{t} \\
& \tilde{h}_{t+1} \equiv h_{t+1}-\xi_{t+1}^{v} J_{t+1}^{v}=\alpha+\delta\left(\tilde{h}_{t}+\xi_{t}^{v} J_{t}^{v}\right)+\sqrt{\tilde{h}_{t}+\xi_{t}^{v} J_{t}^{v}} u_{t} .
\end{aligned}
$$


Since

$$
\begin{aligned}
p\left(\tilde{h}_{t} \mid h_{t-1}, \tilde{h}_{t+1}, \xi_{t}^{v} J_{t}^{v}, \xi_{t+1}^{v} J_{t+1}^{v}\right) & =\frac{p\left(\tilde{h}_{t}, h_{t-1}, \tilde{h}_{t+1}, \xi_{t}^{v} J_{t}^{v}\right)}{p\left(h_{t-1}, \tilde{h}_{t+1}, \xi_{t}^{v} J_{t}^{v}, \xi_{t+1}^{v} J_{t+1}^{v}\right)} \\
& =\frac{p\left(\tilde{h}_{t+1} \mid \tilde{h}_{t}, \bullet\right) p\left(\tilde{h}_{t} \mid h_{t-1}, \bullet\right) p\left(h_{t-1}\right)}{p\left(\tilde{h}_{t+1} \mid h_{t-1}, \bullet\right) p\left(h_{t-1}\right)} \\
& \propto p\left(\tilde{h}_{t+1} \mid \tilde{h}_{t}, \bullet\right) p\left(\tilde{h}_{t} \mid h_{t-1}, \bullet\right) .
\end{aligned}
$$

Thus we have the posterior of $\tilde{h}_{t}$

$$
\begin{aligned}
& p\left(\tilde{h}_{t} \mid h_{t-1}, \tilde{h}_{t+1}, \xi_{t}^{v} J_{t}^{v}, \xi_{t+1}^{v} J_{t+1}^{v}\right) \\
& \propto\left(\tilde{h}_{t}+\xi_{t}^{v} J_{t}^{v}\right)^{-1} \exp \left\{-\frac{1}{2}\left[\operatorname{tr}\left(\sum^{*-1} \tilde{e}_{t} \tilde{e}_{t}^{\prime}\right)+\operatorname{tr}\left(\sum \tilde{e}_{t+1} \tilde{e}_{t+1}^{\prime}\right)\right]\right\} \\
& \propto\left(\tilde{h}_{t}+\xi_{t}^{v} J_{t}^{v}\right)^{-1} \exp \left\{-\frac{\left[\tilde{h}_{t}-\left(\alpha+\delta h_{t-1}+\psi\left(\tilde{r}_{t}-d\right)\right)\right]^{2}}{2 \Omega h_{t-1}}-\frac{\left(\tilde{r}_{t+1}-d\right)^{2}}{2\left(\tilde{h}_{t}+\xi_{t}^{v} J_{t}^{v}\right)}\right. \\
& \left.\quad-\frac{\delta^{2}}{2 \Omega\left(\tilde{h}_{t}+\xi_{t}^{v} J_{t}^{v}\right)}\left[\tilde{h}_{t}+\xi_{t}^{v} J_{t}^{v}-\frac{1}{\delta}\left[\tilde{h}_{t+1}-\alpha\right]\right]^{2}+\frac{\psi}{\Omega} \frac{\left(\tilde{h}_{t+1}-\alpha\right) \tilde{r}_{t+1}}{\tilde{h}_{t}+\xi_{t}^{v} J_{t}^{v}}\right\} .
\end{aligned}
$$

The posterior of $\xi_{t}^{v}$ can be derived from the following relation:

$$
p\left(\xi_{t}^{v} \mid \xi_{t}^{r}, r_{t}, h_{t}, J_{t}=1\right) \propto p\left(r_{t}, h_{t} \mid \xi_{t}^{v}, \xi_{t}^{r}, J_{t}=1\right) p\left(\xi_{t}^{v} \mid u_{v}, J_{t}=1\right)
$$

We know that $p\left(r_{t}, h_{t} \mid \theta, h_{t-1}, \xi_{t}^{v}, \xi_{t}^{r}\right) \propto h_{t-1}^{-1}\left|\sum\right|^{-1 / 2} e^{-\frac{1}{2} \operatorname{tr}\left(\sum^{-1} e_{t} e_{t}^{\prime}\right)}, e_{t}=$ $\left(\varepsilon_{t}, u_{t}\right)^{\prime}$. As a result,

$$
\begin{aligned}
& p\left(\xi_{t}^{v} \mid \xi_{t}^{r}, r_{t}, h_{t}, J_{t}=1\right) \\
& \propto p\left(r_{t}, h_{t} \mid \xi_{t}^{v}, \xi_{t}^{r}, J_{t}=1\right) p\left(\xi_{t}^{v} \mid u_{v}, J_{t}=1\right) \\
& \propto\left\{e^{-\frac{1}{2} \operatorname{tr}\left(\sum^{-1} e_{t} e_{t}^{\prime}\right)}\right\}\left\{e^{-\frac{\xi_{v}}{u_{v}}}\right\} \\
& \propto \exp \left\{-\frac{1}{2} \frac{1}{\Omega h_{t-1}}\left[\xi_{t}^{v}-\left(h_{t}-\alpha-\delta h_{t-1}-\psi\left(r_{t}-d-\xi_{t}^{r}\right)\right)\right]^{2}\right\} \exp \left\{-\frac{\xi_{v}}{u_{v}}\right\} \\
& \propto \exp \left\{-\frac{1}{2 V_{t, 2}}\left[\xi_{t}^{v}-\left(d_{t, 2}^{v}-V_{t, 2}^{v} / u_{v}\right)\right]^{2}\right\}
\end{aligned}
$$

where $V_{t, 2}^{v}=\Omega h_{t-1}, d_{t, 2}^{v}=h_{t}-\alpha-\delta h_{t-1}-\psi\left(r_{t}-d-\xi_{t}^{r}\right)$. 
Jow-Ran Chang et al.

Thus the posterior of $\xi_{t}^{v}$ is a truncated normal distribution

$$
p\left(\xi_{t}^{v} \mid \xi_{t}^{r}, r_{t}, h_{t}, J_{t}=1\right) \propto 1_{\left[\xi_{t}^{v}>0\right]} N\left(d_{t, 3}^{v}, V_{t, 2}^{v}\right) .
$$

The mean and variance of the normal distribution is $d_{t, 3}=d_{t, 2}^{v}-V_{t, 2}^{v} / u_{v}$ and $V_{t, 2}^{v}$, respectively.

A similar method can be used to derive the posterior of $\xi_{t}^{r}$ :

$$
\begin{aligned}
& p\left(\xi_{t}^{r} \mid, r_{t}, h_{t}, J_{t}=1\right) \\
& \quad \propto p\left(r_{t}, h_{t} \mid \xi_{t}^{r}, \xi_{t}^{v}, J_{t}=1\right) p\left(\xi_{t}^{r} \mid u_{r}, \sigma_{r}, J_{t}=1\right) \\
& \propto \exp \left\{-\frac{1}{2\left(\Omega h_{t-1}\right) /\left(\psi^{2}+\Omega\right)}\left[\xi_{t}^{r}-\left(r_{t}-d+\frac{\psi}{\psi^{2}+\Omega}\right.\right.\right. \\
& \left.\left.\left.\quad \times\left(h_{t}-\alpha-\delta h_{t-1}-\xi_{t}^{v}\right)\right)\right]^{2}\right\} \exp \left\{-\frac{1}{2} \frac{\left[\xi_{t}^{r}-u_{r}\right]^{2}}{\sigma_{r}^{2}}\right\} \\
& \propto \exp \left\{-\frac{1}{2 V_{t, 2}^{r}}\left(\xi_{t}^{r}-d_{t, 2}^{r}\right)^{2}\right\}
\end{aligned}
$$

where $V_{t, 2}^{r}=\left[\left(\sigma_{r}^{2}\right)^{-1}+\left(\frac{\Omega h_{t-1}}{\psi^{2}+\Omega}\right)^{-1}\right]^{-1}, d_{t, 2}^{r}=V_{t, 2}^{r}\left\{\frac{u_{r}}{\sigma_{r}^{2}}+\left(\frac{\Omega h_{t-1}}{\psi^{2}+\Omega}\right)^{-1}\left[r_{t}-d+\right.\right.$ $\left.\left.\frac{\psi}{\psi^{2}+\Omega}\left(h_{t}-\alpha-\delta h_{t-1}-\xi_{t}^{v}\right)\right]\right\}$.

At time $t$, the probability of whether the jump happened is decided by the odd ratio of the two possible outcomes.

$$
\begin{aligned}
O_{t}^{r} & =\frac{p\left(J_{t}^{r}=1 \mid r_{t}, h_{t}, h_{t-1}, \xi_{t}^{v}, \xi_{t}^{r}\right)}{p\left(J_{t}^{r}=0 \mid r_{t}, h_{t}, h_{t-1}, \xi_{t}^{v}, \xi_{t}^{r}\right)} \\
& =\frac{\lambda_{r}}{\left(1-\lambda_{r}\right)} \exp \left(-\frac{1}{2} \frac{\left\{\left[2\left(r_{t}-d\right)-\xi_{t}^{r}\right] / \sqrt{h_{t-1}}-2 \rho u_{t} / \sigma_{v}\right\}\left\{-\xi_{t}^{r} / \sqrt{h_{t-1}}\right\}}{1-\rho^{2}}\right)
\end{aligned}
$$

where $u_{t}=\left(h_{t}-\alpha-\delta h_{t-1}-\xi_{t}^{v} J_{t}^{v}\right) / \sqrt{h_{t-1}} \cdot J_{t}^{r}$ follows Bernoulli distribution with probability of one equalling $p_{t}^{r}$. It is easy to see $\frac{p_{t}^{r}}{1-p_{t}^{r}}=O_{t}^{r}$, so $p_{t}^{r}=$ $\frac{O_{t}^{r}}{1+O_{t}^{r}}$.

We choose Beta as the prior of $\lambda_{r} \cdot \operatorname{prior}\left(\lambda_{r}\right)=\operatorname{Beta}(a, b) \propto \lambda_{r}^{a-1}(1-$ $\left.\lambda_{r}\right)^{b-1}$. The posterior is $p\left(\lambda_{r} \mid J_{r}\right) \propto p\left(J_{r} \mid \lambda_{r}\right) \operatorname{prior}\left(\lambda_{r}\right) \propto\left[\lambda_{r}^{\sum_{t=1}^{T} J_{t}}(1-\right.$ $\left.\left.\lambda_{r}\right)^{T-\sum_{t=1}^{T} J_{t}}\right] \lambda_{r}^{a-1}\left(1-\lambda_{r}\right)^{b-1} \propto \lambda_{r}^{a^{*}}\left(1-\lambda_{r}\right)^{b^{*}}$ where $a^{*}=a+\sum_{t=1}^{T} J_{t}-1$, $b^{*}=b+T-\sum_{t=1}^{T} J_{t}-1$.

The posterior $u_{r}$ and $\sigma_{r}^{2}$ follows directly from standard regression theory and therefore is omitted. The posterior of jump in state equation can be derived with a similar method. 
The prior of $u_{v}$ is assumed to follow $I G\left(\phi_{v}, \lambda_{v}\right)$. The posterior can thus be written as:

$$
\begin{aligned}
p\left(u_{v} \mid \xi_{t \mid J_{t-1}}^{v}\right) & \propto p\left(\xi_{t \mid J_{t=1}}^{v} \mid u_{v}\right) \operatorname{prior}\left(u_{v}\right) \\
& \propto u_{v}^{I} \exp \left(-\frac{1}{u_{v}}\left(\xi_{i_{1}}^{v}+\xi_{i_{2}}^{v}+\cdots+\xi_{i_{I}}^{v}\right)\right) u_{v}^{-\left(\phi_{v}+1\right)} \exp \left(-\frac{\lambda_{v}}{u_{v}}\right) \\
& \propto u_{v}^{-\left(\phi_{v}+I+1\right)} \exp \left(-\frac{\lambda_{v}+\sum_{t=1}^{I} \xi_{i_{t}}^{v}}{u_{v}}\right) .
\end{aligned}
$$

Thus the posterior of $u_{v}$ follows the distribution of Inverse Gamma distribution, $I G\left(\phi_{v}+I, \lambda_{v}+\sum_{t=1}^{I} \xi_{i_{t}}^{v}\right)$.

\section{Empirical Results}

The samples we use are daily change rates of Thai Baht from October 1, 1996 to December 10, 2003, totalling 1877 observations. Our sample periods cover the Asian financial crisis, so the episodes of market crashes can be scrutinized by various jump models. Figure 1 plots the daily change rate of the Thai Baht. It can be seen from the plot that the volatility of different periods is dramatically different. For example, periods before May 1997, seldom have daily change rates of more than $1 \%$. It becomes very common when time moves alone. Also another observation is that extreme values play an important role in our time series. Some data points are significantly larger than others.

The empirical investigation of Thai Baht jump behavior starts from Merton's (1976) Independent Jump Model.

$$
r_{t+1}=u+\sigma \varepsilon_{t+1}+\zeta_{t+1} J_{t+1}
$$

where $\varepsilon_{t+1}$ follows standard normal distribution, and $\zeta_{t+1}$ is a normal random variable with mean $\zeta_{0}$ and variance $\sigma_{\zeta}^{2}$. Here $\zeta_{t+1}$ represents the observations that are rare but large in quantity and $\sigma_{\zeta}^{2}>\sigma^{2}$. In other words, the model assumes the change rate of foreign exchange is mixed by two different normally distributed random variables. If Thai Baht has any jump behavior, the estimation result of this model should give us a basic understanding of the jumping behavior. Table 2 summarizes the MCMC estimation result of Merton's Independent Jump model. The estimation converges quickly, so the statistics are calculated from the last 2,000 sweeps, and the first 1,000 sweeps are discarded. The model divides the Thai Baht daily change rate into two groups: the first is low-jump group with mean of $u=-0.0019$ and standard deviation $\sigma=0.316$; 
the second is high-jump group with mean equaling $\zeta_{0}+u=0.145$ and standard deviation $\sqrt{\sigma_{\zeta}^{2}+\sigma^{2}}=2.2$. The probability of a high jump is $\lambda=0.138$. Note that the sample standard deviation is 0.926 in Table 3 , so the majority of the exchange rate changes (the low-jump group) are much smaller than the sample standard deviation. Thus, the estimation results suggest that much of the sample standard deviation is contributed by only a small sample of observations, which have bigger-than-usual daily change rate.

Though Merton's Independent Jump model tells us that part of the daily change rate of Thai Baht is substantially larger than others, the model itself has some unreasonable results. The probability of having a high-jump observation, $\lambda$, is 0.138 , which means that more than 30 trading days in a year have high-jump values. Common sense tells us that the frequency is too high. One reason for this unreasonable high jump frequency is that the model does not consider the well known heteroskedasticity effect in exchange rate time series. To account for the conditional heteroskedasticity effect, we first estimate a square root stochastic volatility model that is reported in Table 1.

Like the estimation of UK Sterling/US Dollar, German Deutschemark/US Dollar, Japanese Yen/US Dollar, and Swiss Franc/US Dollar (Kim and Chib, 1995), the Thai Baht/US Dollar time series have high persistence variance $(\delta=0.993)$. The leverage effect is small $(\rho=-0.012)$, which is similar to the behavior of UK Sterling and Deutschemark (Jacquier, Polson and Rossi, 2003). However, the estimation is biased. It can be seen easily from at least three points. First, the expected estimate value volatility is 0.637 , which is much smaller than the sample variance of 0.857 . Second, before the July 2, 1997 depression, estimated volatility continued to raise from April 17th for 53 days. There were no signs of abnormal behaviors in the exchange market that can be connected to the raise of volatility in April. Third, the estimated volatility in July 2 is 5.261 , which means that the movement of $17 \%$ is a realization of more than 7 standard deviations. The probability of such an event is so rare that it is not too bold to interpretate it as some kind of estimation bias.

One way to correct the estimation bias of square root stochastic volatility is by using log stochastic volatility model. The log stochastic volatility model has a similar structure to the square root stochastic volatility model. But is more flexible due to the log transformation in the transition equation. The $\log \mathrm{SV}$ model used in our research is:

$$
\begin{gathered}
r_{t}=d+\sqrt{h_{t}} \varepsilon_{t} \\
\ln h_{t}=\alpha+\delta \ln h_{t-1}+u_{t} .
\end{gathered}
$$


The Jump Behavior of Foreign Exchange Market: Analysis of Thai Baht • 277

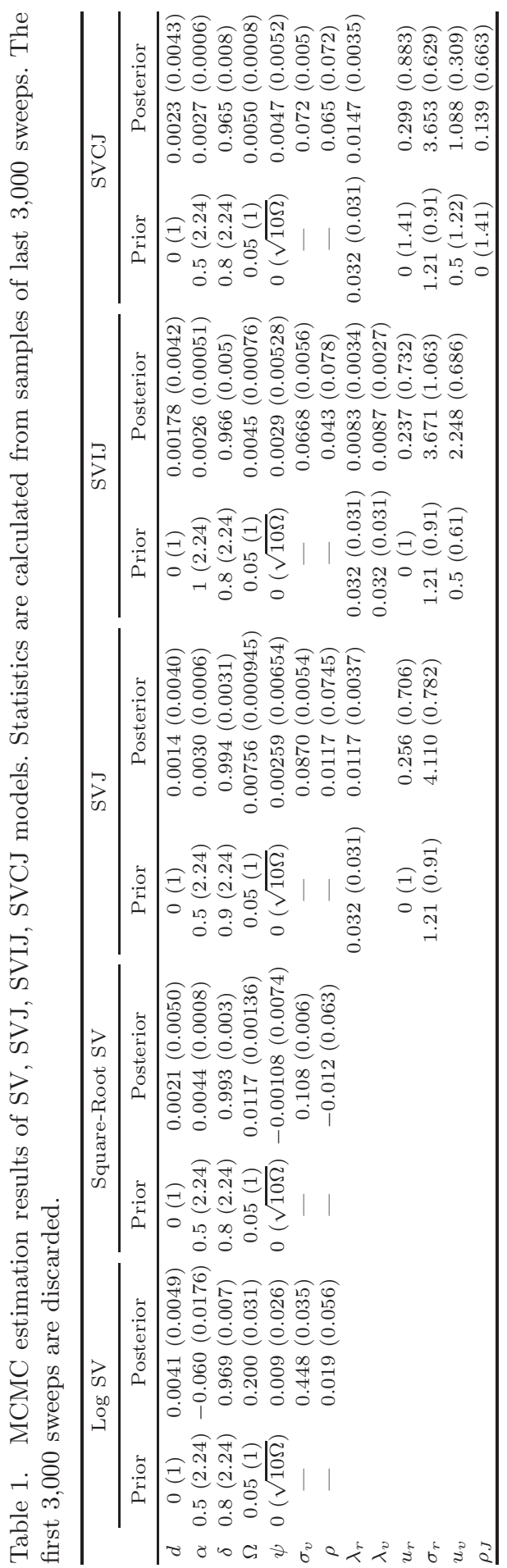


Table 2. Merton's independent jump model. $r_{t+1}=u+\sigma \varepsilon_{t+1}+\zeta_{t+1} J_{t+1}$ $\varepsilon_{t+1} \sim N(0,1), \zeta_{t+1} \sim N\left(\zeta_{0}, \sigma_{\zeta}^{2}\right), J_{t+1} \sim \operatorname{Ber}(\lambda), t=1, \ldots, T$. Statistics calculate from last 2,000 sweep off MCMC samples. The first 1,000 sweeps are discarded.

\begin{tabular}{lccccc}
\hline & $u$ & $\sigma$ & $\zeta_{0}$ & $\sigma_{\zeta}$ & $\lambda$ \\
\hline Prior & $0(5)$ & $0.87(0.32)$ & $0(5)$ & $1.74(0.63)$ & $0.032(0.031)$ \\
Posterior & $-0.0019(0.0092)$ & $0.316(0.010)$ & $0.147(0.126)$ & $2.197(0.106)$ & $0.138(0.010)$ \\
\hline
\end{tabular}

Table 3. Descriptive statistics of Thai Baht daily change rate.

\begin{tabular}{lccccc}
\hline & Mean & Std. Dev & Skewness & Kurteness & Range \\
\hline Daily change rate of Thai Baht & 0.024 & 0.926 & 70.39 & 3.03 & 23.24 \\
\hline
\end{tabular}

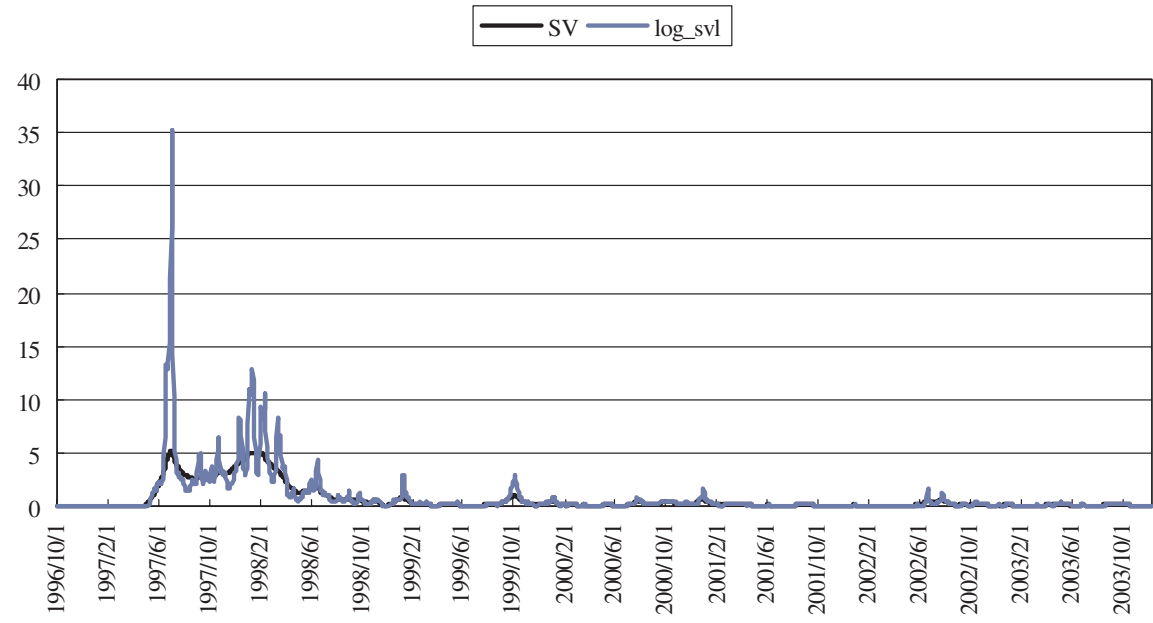

Fig. 2. Estimated volatility between Square Root SV (SV) and Log SV (log_svl) model.

The residual of state and transition equation have the same covariance structure as the square root SV model. Figure 2 compares the estimation results of the two models. The log SV model is apparently more flexible since it responds well to the volatile movement of the foreign exchange market on July 2 - a soaring volatility of 35.16. The movement of July 2 is a realization of less than three standard deviations. Though such an event is still very rare, it is much more reasonable compared to the estimation result of the Square Root SV model. Similar correction can be found in other days when the foreign exchanges experience unusually large movements. Moreover, from Table 4, we can see that the mean of the estimated volatility is 0.807 , which is close to the sample variance. Despite the favorable outcomes 
Table 4. Some description statistics of estimated volatility.

\begin{tabular}{lccc}
\hline & Mean & Std. Dev. & Variance Caused by Jump in Obs. \\
\hline Log SV & 0.807 & 2.131 & - \\
Square root SV & 0.637 & 1.139 & - \\
SVJ & 0.502 & 0.889 & $42 \%$ \\
SVIJ & 0.654 & 1.434 & $34 \%$ \\
SVCJ & 0.538 & 1.048 & $37 \%$ \\
\hline
\end{tabular}

mentioned above, the Log SV model still shows signs of estimation bias. Like the Square Root SV, the estimated volatility starts to rise around mid-April, a fact suggesting the influence of extreme value.

Extreme value must be coped with in order to have a correct estimation. The first model we use is the Square Root SV model with jump in the observation equation.

$$
\begin{aligned}
& r_{t}=d+\sqrt{h_{t-1}} \varepsilon_{t}+\xi_{t}^{r} J_{t}^{r} \\
& h_{t}=\alpha+\delta h_{t-1}+\sqrt{h_{t-1}} u_{t} .
\end{aligned}
$$

The transition equation allows gradual evolution of volatility. Anything that does not fit into this pattern will be interpreted as jump at return. The estimation results are summarized in Table 1. Like the Log SV or the Square Root SV model, the volatility persistence is very high. The jump frequency is 0.0117 , which roughly rounds up to three times per year. The mean of the jump component is 0.256 , which is not significantly different from zero. The standard deviation of the jump component is 4.11 , which is quite large compare to the sample variance. On July 2, it was 15.192, which shows that about $90 \%$ of the total movement is attributed to the jump component. In our whole sample, the SVJ model attributes $42 \%$ of its sample variance to the jump component of the observation equation. Compared to Merton's Independent jump model, only a very small fraction of observations are classified as jumps. The frequency of 3 jumps per year is closer to our common sense that jump is a rare and large movement.

The SVJ model still has trouble catching up with the movement of the Thai Baht in Fig. 3. Estimated volatility still shows patterns of consecutive increase from late April to July 2. The pattern of consecutive increase in estimated volatility suggests that the state equation needs to be relaxed so that it can catch up to the foreign market's movement. Figure 3 compares the estimated volatility of Square Root SV and SVJ models. Removing 


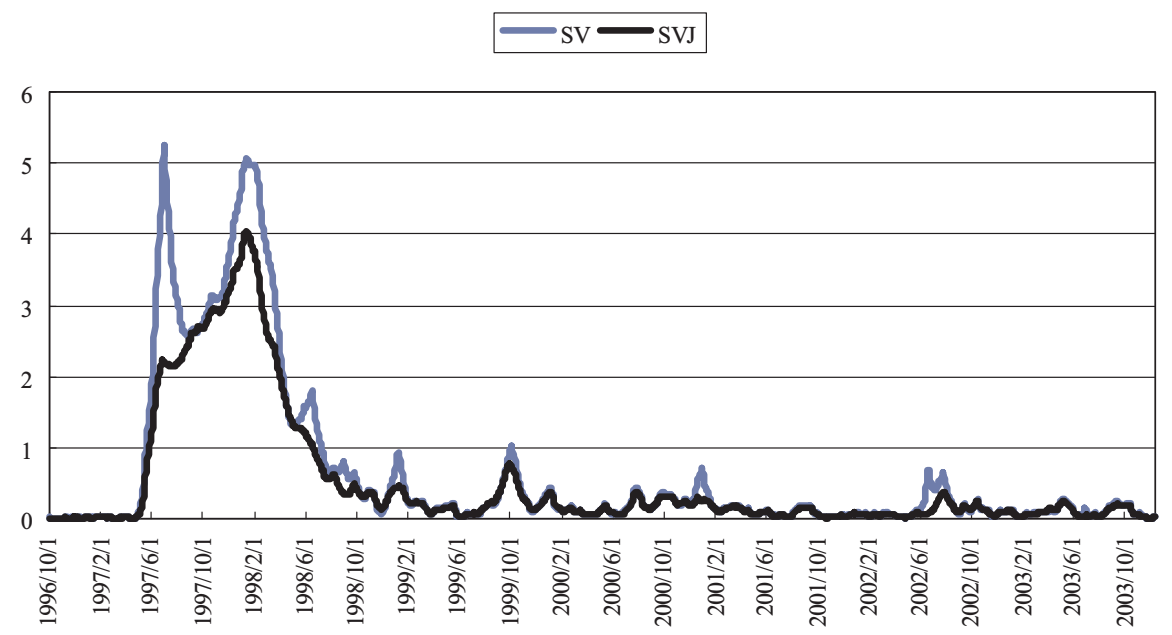

Fig. 3. Estimated volatility between SV and SVJ model.

extreme value by the jumping component at observation reduces the estimated volatility at various periods. Figure 5 presents the estimated jump under the SVJ model.

Further relaxation can be made by introducing another jump component in the state equation. The model becomes

$$
\begin{aligned}
& r_{t}=d+\sqrt{h_{t-1}} \varepsilon_{t}+\xi_{t}^{r} J_{t}^{r} \\
& h_{t}=\alpha+\delta h_{t-1}+\sqrt{h_{t-1}} u_{t}+\xi_{t}^{v} J_{t}^{v}
\end{aligned}
$$

The above model is a Square Root Stochastic Volatility model with Independent Jump in the observation and state equations (SVIJ). The estimation result of SVIJ can be found in the third column of Table 1. The volatility persistence is down to 0.966 while the jump component of the observation equation has similar estimated mean and variance. Average jump size volatility is 0.237 . The jump frequency in observation and state equations are similar, roughly about 2.3 times per year. The mean of the estimated volatility is 0.654 , which means SVIJ model attributes $34 \%$ of its sample variance to the jump component in the observation equation. The estimated volatility does not increase gradually in April. Instead, it has a jump of 2.20 in May 13, the week when speculators were attacking Thai Baht furiously. Unlike the previous models, the estimation result of SVIJ shows no sign of bias. Figure 4 compares the estimated volatility between SV and SVIJ models. SVIJ model responses to the increased volatility by sharply raising the 


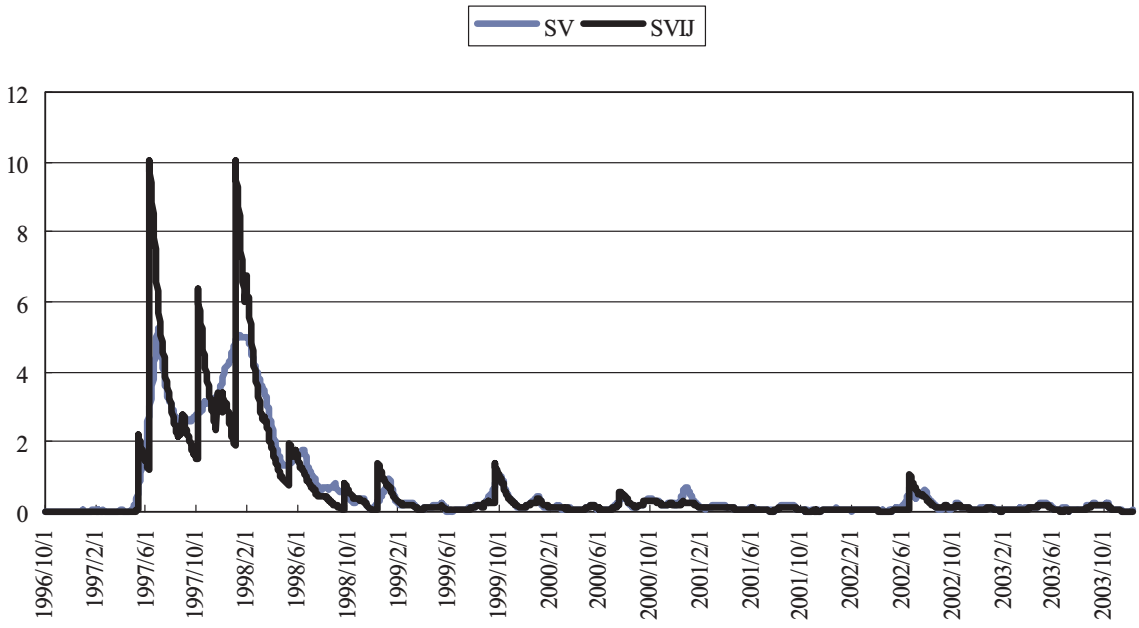

Fig. 4. Estimated volatility between SV and SVIJ model.

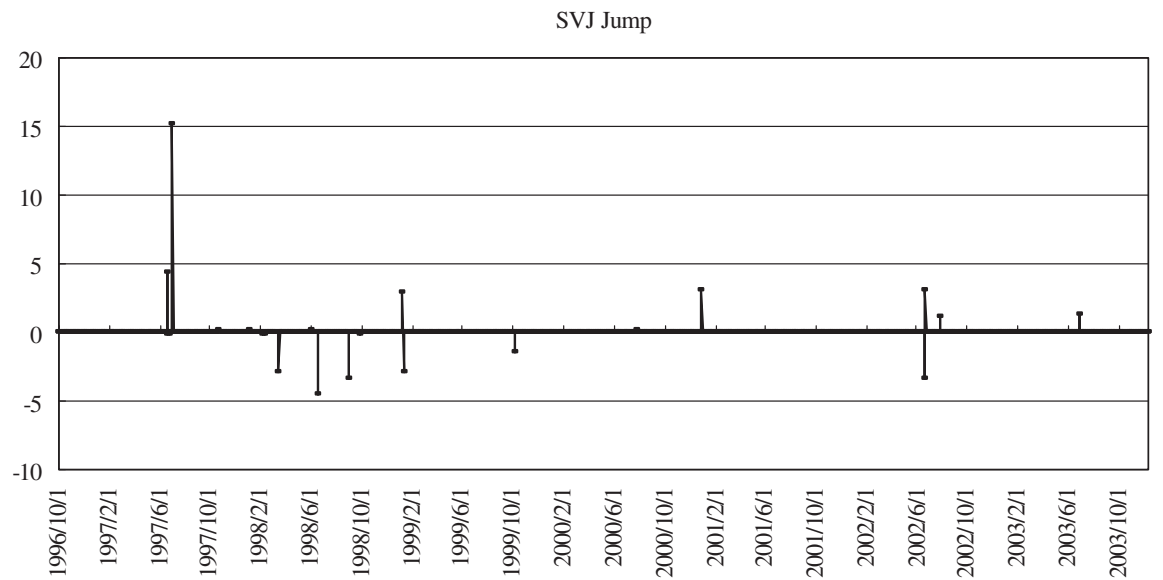

Fig. 5. Jump estimation of SVJ model.

estimated volatility. Figures 6 and 7 present the estimated jump in foreign exchange and its volatility, respectively.

The SVCJ model restricted the jump at observation and state equation to happen simultaneously. The estimation result is similar to the SVIJ model. The volatility persistence is 0.965 and the leverage effect is near zero. The jumping frequency is 0.0147 , which roughly equals to 3.8 times per year. Compared to the SVIJ model, the jump at observation has a standard deviation of similar size, but the SVCJ estimated a larger jump size in the state equation. Figure 8 compares the estimated volatility between SV 
SVIJ Jump at observation equation

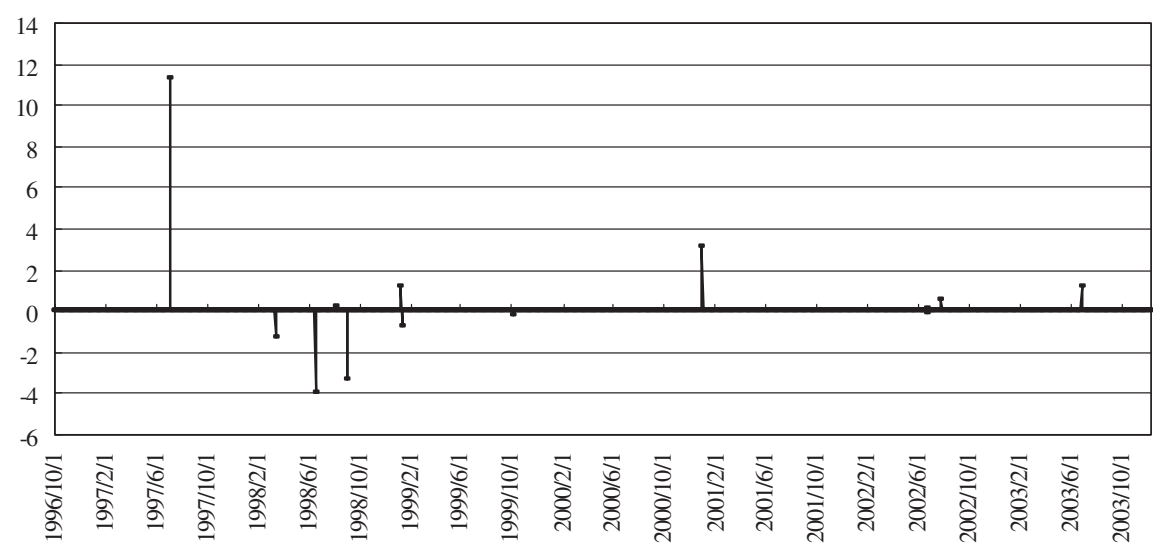

Fig. 6. Estimated jump at observation equation of SVIJ model.

SVIJ Jump at Volatility

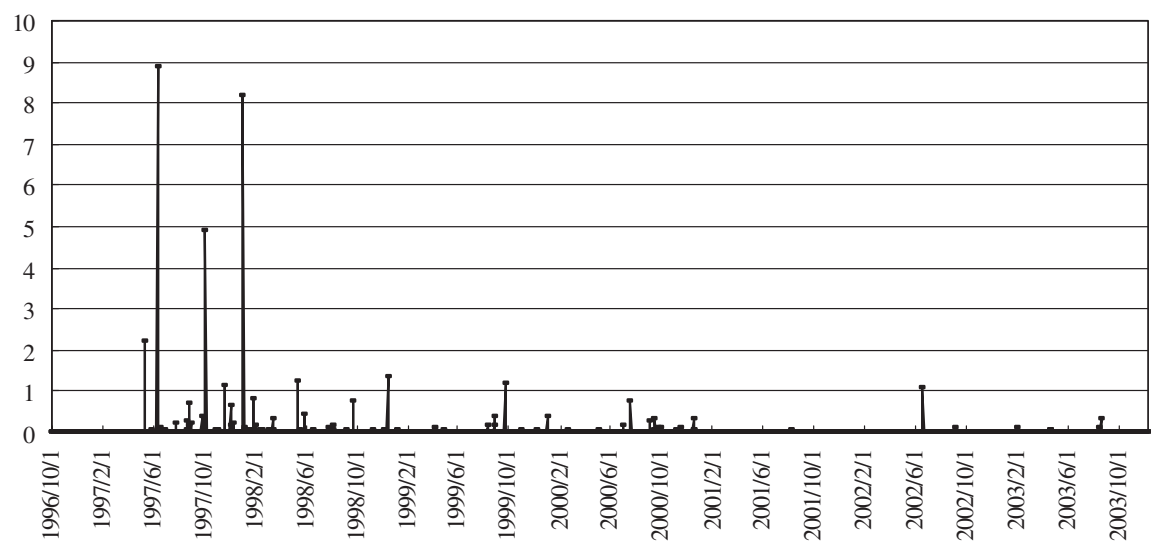

Fig. 7. Estimated volatility jump of SVIJ model.

and SVCJ models. The SVCJ model responds to the increased volatility by sharply raising the estimated volatility. Figures 9 and 10 present the estimated jump in a foreign exchange and its volatility, respectively. A summary of estimations can be found in the last column of Table 1.

\section{Model Diagnosis and Bayesian Factor}

Following Eraker, Johannes and Polson (2003), we use Bayesian factor to evaluate the explanatory power of SV, SVJ, SVCJ and SVIJ models. Log SV 


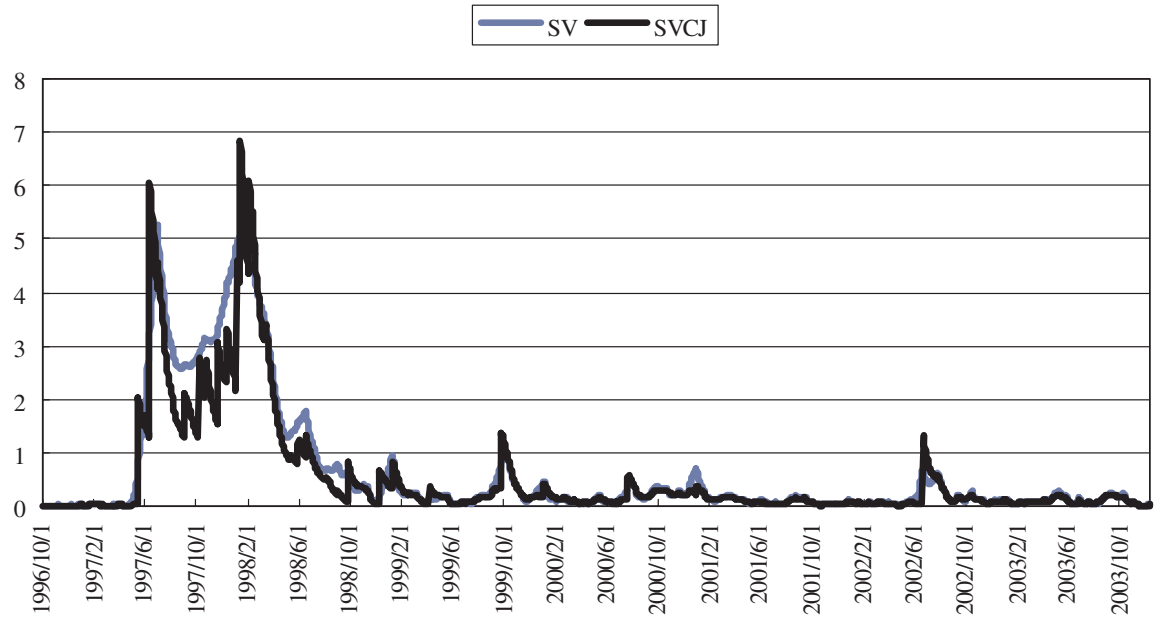

Fig. 8. Compare the estimated volatility between SV and SVIJ model.

Jump at observation equation (SVCJ)

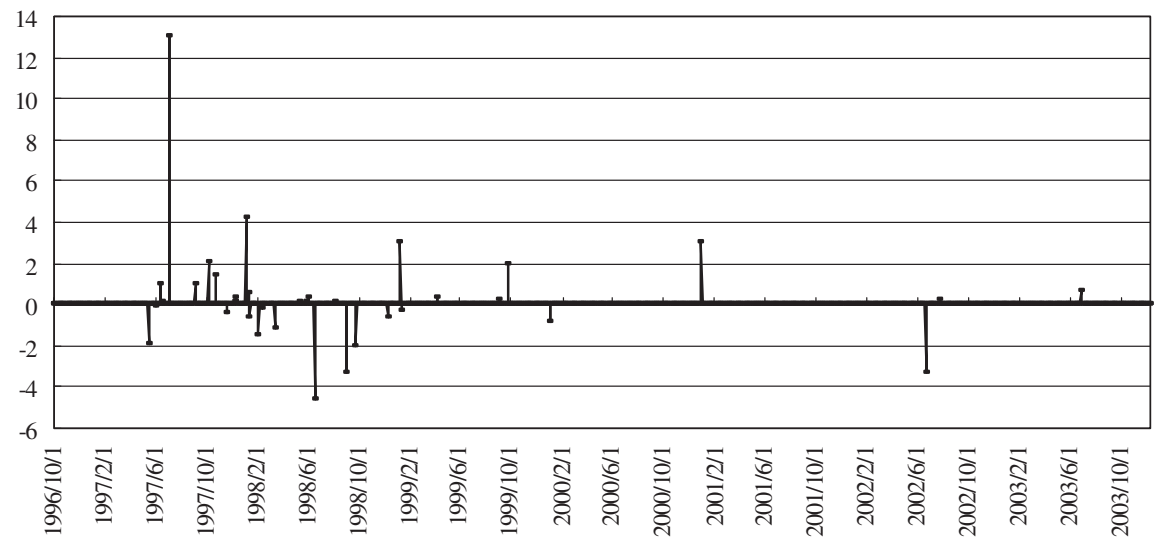

Fig. 9. Estimated jump at observation equation of SVIJ model.

and Merton's independent jump model is excluded because they serve only for comparison purpose. Unlike the likelihood ratio test, which evaluates the likelihood function of two competing models at the point which maximizes the likelihood function, Bayesian factor calculates the posterior ratio of two likelihood function by integrating out other parameters. Thus Bayesian factor takes parameter uncertainly into consideration. Detail discussion can be found in Kass and Raftery (1995). 
Jump at Volatility (SVCJ)

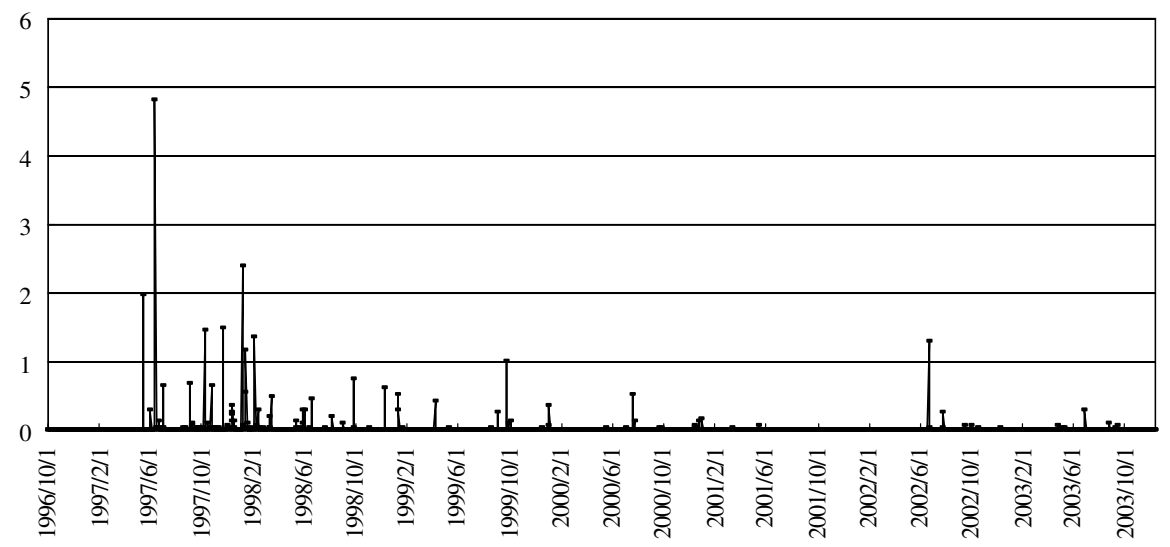

Fig. 10. Estimated volatility jump of SVIJ model.

The Bayesian factor between SVJ and SV is calculated by the following relation:

$$
\frac{p(S V J \mid r)}{p(S V \mid r)}=\frac{p(r \mid S V J)}{p(r \mid S V)} \frac{p(S V J)}{p(S V)}
$$

where $p(S V J \mid r)$ represents the probability that SVJ is the true model after observations are given and $p(S V \mid r)$ represents the probability of having $\mathrm{SV}$ model as the true model. The ratio $\frac{p(S V J)}{p(S V)}$ is the prior probability ratio of these two models. If we do not have a preference for either of them, then the ratio of the two likelihood function $\frac{p(r \mid S V J)}{p(r \mid S V)}$ is a Bayesian factor. The calculation of the Bayesian factor can be found in Eraker, Johannes and Polson (2003).

According to Kass and Raftery (1995), log Bayesian factor between zero and one suggests that the two models have no difference; log Bayesian factor between one to three suggests that the competing model is better than the base model; three to five suggests strong evidence against base model; log Bayesian factor larger than 5 is a solid evidence in favor of the competing model. Moreover, Bayesian factor can be converted to normal $t$-value. With a sample size of one thousand, log Bayesian factor one is equivalent to the value of 2.63; two equivalent to 3.59 ; and three equivalent to 4.11 .

Table 5 summarizes the Bayesian factor between SV, SVJ, SVIJ, and SVCJ. SVJ, SVIJ and SVCJ all have better explanatory powers than the SV model. SVCJ beats SVJ model easily, and it is beaten by SVIJ. Thus 
Table 5. Summary of log Bayesian factor.

\begin{tabular}{cccccc}
\hline & SVJ vs SV & SVIJ vs SV & SVIJ vs SVJ & SVCJ vs SV & SVIJ vs SVCJ \\
\hline Bayesian factor & 19.90 & 34.71 & 10.13 & 21.18 & 13.53 \\
\hline
\end{tabular}

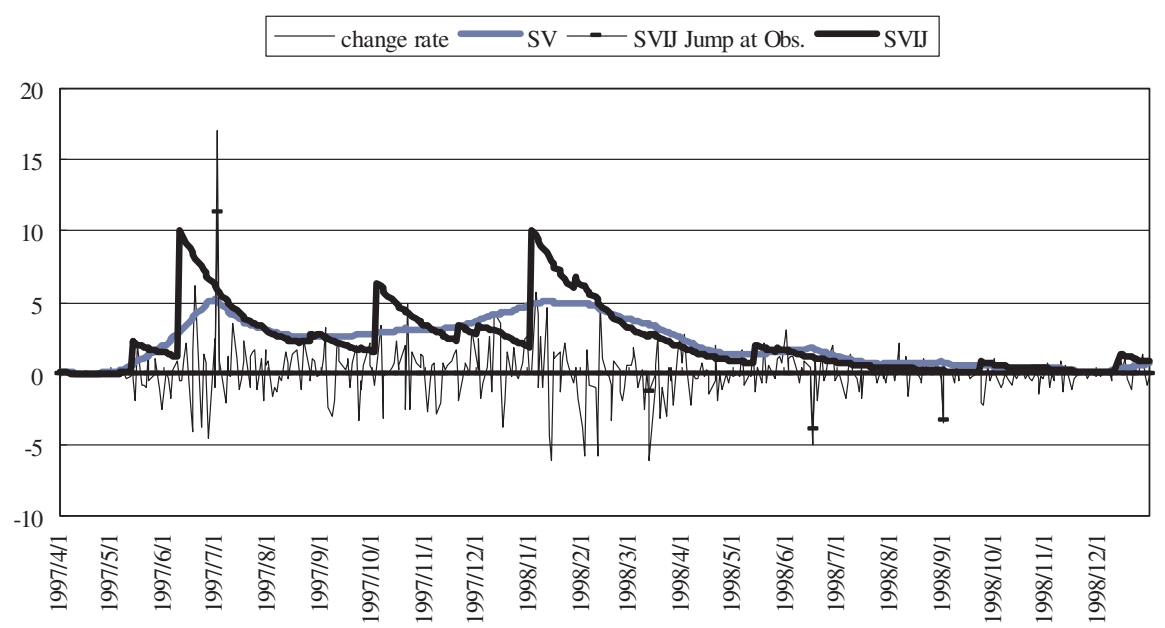

Fig. 11. The volatility estimated by SV and SVIJ model, jump at observation of SVIJ model. From 1997/4/1 to 1998/12/31.

the SVIJ model has the best explanatory power among the four competing models we considered.

\section{Matching Events and Estimation Results}

The historical price of Thai Baht can be seen as a result of the interactions between the Thai government, market speculators, and other counterparts in the foreign exchange market. While the players in the foreign exchange market obviously have different goals, the estimation results in the previous sections can be used to gain a better understanding of how different market players affect the behavior of the Thai Baht. We use the chronology of the Asian financial crisis composed by Nourial Roubini ${ }^{1}$ and a summary of Thailand's currency crisis by Ito (1999) for the major sources of events. Since SVIJ and SVCJ have similar results and SVIJ is a better explanatory model, we will focus on the results of the SVIJ model in this section. Figure 11 presents a detailed plot of SVIJ estimation results during the period from April 1997 to December 1998.

\footnotetext{
${ }^{1}$ Available from internet: http://www.stern.nyu.edu/globalmacro/.
} 
Before and in May, 1997

Thai Baht attracted the attack of speculators in 1997 because of the country's slow GDP growth rate and unstable financial institutions. Several waves of speculative attack took place in January, February and May 1997. However, from the estimation results of SV family models, there are no signs that Thai Baht was affected by these attacks. When May came along, the situation became different. The waves of attacks that began on May 12 sharply raised the estimated volatility of SVIJ model to 2.24 from 0.04 . The model identified a jump in volatility during a particular period, which suggests that the Thai government failed to maintain the stability of its foreign exchange market, and therefore had lost in this battle.

June - July, 1997

While the speculators continue to attack, another jump in volatility was identified on June 11. The scale was of 8.873, thus pushing the estimated volatility to 10.05. This jump declared the beginning of a more volatile episode. Daily change of more than $3 \%$ became common during this period. For example, Thai Baht appreciated $4.15 \%$ on June 17, depreciated $6.19 \%$ on June 19, appreciated $4.54 \%$ on June 27 , and finally deprecated $17 \%$ on July 2. The SVIJ model estimated a jump on the observation equation of $11.3 \%$ on July 2 , and attributes the rest of the $35 \%$ changes to the conditional volatility parts of the model.

While the Thai Baht continued to slide after July 2, the estimated volatility declined gradually, with the exception of a few sporadic occasions. The next major volatility jump happened on October 6 while Thai Baht depreciated $3.43 \%$ and appreciated $3.15 \%$ the next day. The SVIJ model identified this as a jump of volatility of size 4.9 .

\section{January - June 1998}

In January 5, 1998, Thai Baht depreciated $5.75 \%$ and $4.13 \%$ on the next day. In the next few days, the foreign exchange rate fluctuated heavily in response to Stand \& Poor's announcement on December 31, 1997 that Indonesia's sovereign rating was being downgraded. It caused a bearish sentiment in Southeast Asian countries, including Thailand. With this particular event, the SVIJ model identified a jump of 8.18 in volatility on January 5, 1998. Volatility during this period was about 9.5 , which meant a change of $3 \%$ to $4 \%$ was common. After reaching the record low of 56.09 to one dollar on January 12, 1998, the Thai Baht started to appreciate. In the meanwhile, 
the volatility declined gradually until the next volatility jump on May 14, 1998. The volatility jumped from 0.76 to 1.95 in response to the worsening situation in Indonesia.

Two occurrences of jump in observation equation have been identified in the periods from January to June 1998. The first occurrence was on March 13, 1998 when the foreign exchange market responded to a large stock market gain in Japan and other countries in Southeast Asia. The Thai Baht appreciated $6.17 \%$ that day. The second jump happened on June 17, 1998, which was affected by the appreciation of the Japanese Yen and Singapore dollar. The Thai Baht appreciated $5.05 \%$ while $80 \%$ of the change was identified as jump in observation equation. These market changes were identified as jump in observation equation because they were single events no sign of volatility change before or after that particular day.

\section{July 1998 - December 2003}

The volatility continued to decline after the jump on May 14, 1998, and reached 0.0072 on September 23, 1998. On September 24, another wave of active trading in the foreign exchange pushed the volatility up to 0.847 . After that, only sporadic events on December 16, 1998, September 23, 1999, and June 21, 2002 had small scale volatility jumps. The decline of volatility clearly announced the end of the Asian financial crisis. Several jump in observation happened during this time. The model identified September 1, 1998, January 11, 1999, December 29, 2000, and June 27, 2003 as having jump in observation.

\section{Conclusion}

The behavior of Thai Baht from October 1996 to December 2003 is analyzed in this paper. The estimation result of the SV model with jump confirms our suspicion that there is jump behavior in the foreign exchange market of the Thai Baht. Ignoring the jump component in the time series model will cause bias estimation results. Bayesian factor, used as a model diagnosis tool, indicates that among the competing models, SVIJ has the best explanatory power. While trying to match jumping behavior to major events, it turns out that SV with jump models do correspond with jump in observation or volatility during these episodes. For example, while the foreign exchange market was attached by speculators in May 1997, the SVIJ and SVCJ models corresponded well with the jump in volatility. Also, jump in observation is common in periods of market stress. 
Dynamic volatility model is useful for derivatives pricing, futures hedging, risk management and regulation for investors, currency trader and central bankers. The study in this paper delivers a clear message that jumping components, both in observation and state equations, are important in the foreign exchange markets. Any modeling attempt for foreign exchange time series should consider the possibility that jumping behavior exist in the interest of time series.

\section{References}

Bakshi, G, C Cao and Z Chen (1997). Empirical performance of alternative option pricing models. Journal of Finance, 52, 2003-2049.

Bates, D (1996). Jumps and stochastic volatility: Exchange rate processes implicit in Deutsche mark options. Review of Financial Studies, 9(1), 69-107.

Bjork, T, Y Kabanov and W Runggaldier (1997). Bond market structure in the presence of marked point processes. Mathematical Finance, 7(2), 211-239.

Chib, S, F Nardari and N Shephard (2002). Markov chain Monte Carlo methods for stochastic volatility models. Journal of Econometrics, 108, 281-316.

Das, S (1998). A direct discrete-time approach to Poission-Gaussian bond option Pricing in the Health-Jarrow-Morton Model. Journal of Economic Dynamics and Control, 23, 333-369.

Duffie, D and R Kan (1996). A yield-factor model of interest rates. Mathematical Finance, 6(4), 379-406.

Eraker, B (2002). Do equity prices and volatility jump? Reconciling evidence from spot and option prices. Working Paper, Duke University.

Eraker, B, M Johannes and N Polson (2003). The impact of jumps in volatility and returns. Journal of Finance LVIII, 1269-1299.

Ito, T (1999). The development of the Thailand currency crisis: A chronological review. NBER Session on Thailand, April 22, 1999.

Jacquier, E, N Polson and P Rossi (1994). Bayesian analysis of stochastic volatility models. Journal of Economics and Business Statistics, 12, 371-389.

Jacquier, E, N Polson and P Rossi (2004). Bayesian analysis of stochastic volatility models with fat-tailed and correlated errors. Journal of Econometrics, 122, $185-212$.

Kass, RE and AE Raftery (1995). Bayesian factors. Journal of the American Statistical Association, 90, 773-795.

Merton, R (1976a). Option pricing when the underlying stock returns are discontinuous. Journal of Financial Economics, 3, 125-144.

Merton, R (1976b). The impact on option pricing of specification in the underlying stock price returns. Journal of Finance, 31, 333-350.

Venkataraman, S (1997). Value at risk for a mixture of normal distributions: The use of quasi-Bayesian techniques. Federal Reserve Bank of Chicago Economic Perspectives, 21(2), 2-13.

Zangari, P (1996). An improved methodology for measuring VAR. RiskMetrics Monitor, 2, 7-25. 